\title{
IN-SITU SENSOR-BASED DAMAGE DETECTION OF COMPOSITE MATERIALS FOR STRUCTURAL HEALTH MONITORING
}

\author{
Seth S. Kesslerand S. Mark Spearing
}

Department of Aeronautics and Astronautics, Massachusetts Institute of Technology, Cambridge, MA 02139, USA

\begin{abstract}
Structural Health Monitoring (SHM) denotes a system with the ability to detect and interpret adverse "changes" in a structure in order to improve reliability and reduce life-cycle costs. The greatest challenge in designing a SHM system is knowing what "changes" to look for and how to identify them. The characteristics of damage in a particular structure plays a key role in defining the architecture of the SHM system. The resulting "changes," or damage signature, will dictate the type of sensors that are required, which in-turn determines the requirements for the rest of the components in the system. The present research project focuses on the relationship between various sensors and their ability to detect "changes" in a structure's behavior. This paper presents part of an experimental and analytical survey of candidate methods for in-situ damage detection of composite materials using piezoceramic sensors (PZT). Results are presented for the application of modal analysis, Lamb wave, acoustic emission and strain monitoring techniques to quasi-isotropic graphite/epoxy specimens containing representative damage modes, including delamination, transverse ply cracks and through-holes.
\end{abstract}

\section{INTRODUCTION}

Structural health monitoring essentially involves the embedding of an NDE system (or a set of NDE systems) into a structure to allow continuous remote monitoring for damage. There are several advantages to using a SHM system over traditional inspection cycles, such as reduced down-time, elimination of component tear-down and the potential prevention of failure during operation. Aerospace structures have one of the highest payoffs for SHM applications since damage can lead to catastrophic and expensive failures, and the vehicles involved undergo regular costly inspections. Currently $27 \%$ of an average aircraft's life cycle cost, both for commercial and military vehicles, is spent on inspection and repair; a figure that excludes the opportunity cost associated with the time the aircraft is grounded for scheduled

\footnotetext{
* Copyright $\odot 2002$ The American Institute of Aeronautics and Astronautics Inc. All rights reserved
}

maintenance $^{1}$. New military fighter-craft such as the Eurofighter, the Joint Strike Fighter and the F-22 all incorporate Health Usage Monitoring Systems (HUMS), which record peak stress, strain and acceleration experienced in key components of the vehicle ${ }^{2}$. While these measurements provide useful information about the state of the vehicle between flights, the value of such a system could be greatly increased if continuous data could be accessed instantaneously.

As companies strive to lower their operational costs, many SHM schemes have been developed by industry, universities and research institutes. In a collection of papers written by Zimmerman, he suggests that an algorithmic approach could be used to enhance the model correlation and health monitoring capabilities using frequency response methods ${ }^{3}$. Minimum rank perturbation theory is used to address the problem of incomplete measurements, since a true structure does not conform to ideal conditions. Other researchers have developed algorithms to attempt to correlate modal response under arbitrary excitation to models using a probabilistic sub-space based approach ${ }^{4}$. Recently, Boeing has been exploring the use of frequency response methods in SHM systems for composite helicopter blades $^{5}$. Their system, which is called Active Damage Interrogation (ADI), uses piezoelectric actuators and sensors in various patterns to produce transfer functions in components that are compared to baseline "healthy" transfer functions to detect damage. Giurgiutiu used Lamb wave techniques to compare changes in thin aluminum aircraft skins after various levels of usage to detect changes, and used finite element techniques to attempt to predict the level of damage with some success ${ }^{6}$. More detailed work was done by Cawley's group at Imperial College, who used Lamb waves to examine experimentally representative metallic aircraft components such as lap joints, painted sections and tapered thickness ${ }^{7}$. This paper concludes that these methods present good sensitivity to localized damage sites, however the responses are often complicated to interpret, and many limitations exist for the implementation of these methods over large areas. Honeywell and NASA have been working in a collaborative project since the mid-1990's to introduce an acoustic emission-based SHM system into critical military aircraft components ${ }^{8,9}$. This program, which involved the 
monitoring of T-38 and F/A-18 bulkheads, is one of the most thorough examples of a SHM system to date. These experiments were able to demonstrate successfully the collection of fatigue data and triangulation of some cracks from metallic components while in flight, which could then be analyzed post-flight to make decisions about flightreadiness. In another program Northrop had similar success using AE to monitor small aircraft ${ }^{10}$. They suggested using between 100 and 1000 sensors to implement this system in a larger aircraft depending on whether the entire structure is being monitored or just critical components.

The primary goal of SHM is to be ble to replace current inspection cycles with a continuously monitoring system. This would reduce the downtime of the vehicle, and increase the probability of damage detection prior to catastrophic failure. Several parts of SHM systems have been developed and tested successfully, however much work remains before these systems can be implemented reliably in an operational vehicle. The present research attempts to fill some of the gaps remaining in SHM technologies. NDE techniques with the highest likelihood of success were thoroughly examined, including frequency response, Lamb wave, acoustic emission and strain monitoring methods. For each of these methods, an analytical and experimental procedure was followed to optimize the testing parameters and data interpretation. Their strength, limitations and SHM implementation potential were evaluated, and suggested roles for each are presented. The requirement of the other components necessary in an SHM system are described, and recommendations are offered for a structural health monitoring system architecture based on the results of this research.

\section{FREQUENCY RESPONSE METHODS}

\section{Experimental procedures}

The first damage detection methods surveyed during the present research were the frequency response methods. Detailed results for these experiments have been presented in previous papers ${ }^{11-13}$. Narrow rectangular quasi-isotropic $[90 / \pm 45 / 0]_{\mathrm{s}}$ laminates were manufactured of the AS4/3501-6 graphite/epoxy system with various forms of damage introduced to them, including matrix-cracks, delaminations and through-holes. PZT piezoceramic patches were affixed to each specimen using $3 \mathrm{M}$ ThermoBond ${ }^{\mathrm{TM}}$ thermoplastic tape. In order to measure the natural frequencies of the specimen an impedance meter was used, and the mode shapes were deduced used a scanning laser vibrometer. The specimens were excited using the PZT wafers by a 5
V sine chirp signal, which was sent to the piezos through a function generator to drive them between $0 \mathrm{~Hz}$ and 20 $\mathrm{kHz}$. A table comparing the first six natural frequencies and mode-shapes of a control specimen and several other damaged specimens can be found in Table 1. The velocity magnitude responses comparing the control and damaged specimens for a frequency range below $500 \mathrm{~Hz}$ can be found in previously published papers ${ }^{11-13}$.

\section{Finite element model}

A finite element analysis was performed to predict the frequency response of each specimen. Eight-node quadrilateral shell elements were used to model the specimen, and the "Simultaneous Vector Iteration" method was used to calculate the natural frequencies of the system up to $20 \mathrm{KHz}$, and their corresponding mode shapes. A table comparing the first six natural frequencies and mode-shapes of each specimen can be found in Table 2 An example of a transfer function comparison plot between a control model and one with a delamination is shown in Figure 1.

\section{Discussion}

For both the numerical (FE) and experimental results it is evident that all the forms of damage investigated in this study caused detectable changes in the natural frequencies of a simple coupon. These changes are present in each of the lower normal frequencies discovered, and become more pronounced at higher frequencies. Good correlation was found between the model and the experimental results for low frequencies, however coalescing modes at higher frequencies made comparison impractical. For both the results, as published in the literature, a strong correlation existed between relative frequency reduction and the area damaged by a particular mechanism, however it is difficult to draw any conclusions about the criticality of the damage since there is no information regarding the form of the damage or its orientation. Based on these results, it is likely that an observer can discern whether a structure has been damaged by observing its frequency response, however it would be difficult to differentiate reliably between damage types, locations and orientations. This method appears to be appropriate for detecting global changes in stiffness for relatively large structures at a low power and weight cost.

\section{LAMB WAVE METHODS}

\section{Experimental procedures}

The next method explored during the present research examined the utility of using Lamb waves for damage detection. Again, detailed results for this Lamb wave research has been presented in previous papers ${ }^{13-17}$. The experimental procedures followed a building block approach, and the first set of experiments conducted on 
narrow composite coupons presented in the previous section. Both the actuation and the data acquisition were performed using a portable NI-Daqpad ${ }^{\mathrm{TM}} 6070 \mathrm{E}$ data acquisition board, and a laptop running Labview $^{\mathrm{TM}}$ as a virtual controller. A single pulse of 3.5 sine waves under a Hanning window was sent to the driving PZT at $15 \mathrm{kHz}$ to stimulate an $\mathrm{A}_{0}$ mode Lamb wave, and concurrently the strain-induced voltage outputs were recorded by a sensing PZT wafer. The results were compared by performing a Morlet wavelet decomposition centered at the driving frequency ${ }^{18}$. This procedure was also carried out for beam specimens, laminated plates with bonded stiffeners, and a sandwich construction cylinder. The voltage time traces of each of the narrow coupons along with their complementary wavelet decomposition plots can be found in previously published papers ${ }^{13-17}$. A summary comparing the recorded times of flight for can be found in Table 3 . Probably the most significant result of the present research was the "blind test." Four beam specimen were tested, one with a known delamination while of the remaining three specimens it was unknown which contained a disbond and which were undamaged. By comparing the wavelet plots in Figure 2, it was easily deduce that the two undamaged specimens are the ones with much more transmitted energy, while the third specimen (Control C) obviously has the flaw.

\section{Analytical procedure}

Finite element models were created in ABAQUS ${ }^{\mathrm{TM}}$ to represent each of the experiments, using $1 \mathrm{~cm}^{2}$ square shell elements to predict the small changes in time of flight caused by damage. The results were visualized as a movie file to measure the time of flight of the Lamb waves across the specimens and record visual evidence of dispersion. Series of still shots of Lamb waves propagating in the control and damaged models can be found in a previous work ${ }^{13}$. A summary comparing the recorded times of flight for each of the models can be found in Table 4 .

\section{Discussion}

The results from the narrow coupon tests clearly show the presence of damage in all of the specimens; this was made most obvious by comparing the wavelet decomposition plots. The control specimens retained over twice as much energy at the peak frequency as compared to all of the damaged specimens. The loss of energy in the damaged specimens was due to reflected energy and dispersion. Similar effects of damage were observed in each of the built-up composite structure cases. By comparing the stiffened plates with and without a delamination, a reproducible signal was transmitted across each of the intact portions while it was obvious that the signal traveling through the delaminated region was propagating at a different speed. Finally, in the composite sandwich cylinder the impacted region caused severe dispersion of the traveling Lamb wave, which in turn attenuated the received signal further down the tube. Lamb wave techniques have the potential to provide more information than other methods since they are sensitive to the local effects of damage in a material. Similar to frequency response methods, their results are limited at higher frequencies, however their low frequency results should provide sufficient data to identify damage. The disadvantage of Lamb wave methods is that they require an active driving mechanism, and the resulting data can be more complicated to interpret. Overall however, Lamb wave methods have been found to be effective for the insitu determination of the presence and severity of damage in composite materials.

\section{OTHER PIEZO-BASED METHODS}

\section{Experimental procedures}

There are many advantages to using piezoelectric sensors in SHM applications; they are light, can be conformable, use little power and are sensitive to small strains and accelerations. Previous sections of this paper have given a detailed account of the frequency response and Lamb wave methods using piezo sensors. Both of these methods have demonstrated useful sensitivity to damage, however they are most effectively implemented actively by using powered actuators in a pulse-transmission or pulse-echo mode. Perhaps the greatest advantage of using piezoelectric material for sensors, is that they can be used for a wide variety of detection techniques by simply altering the time scale of analysis or actuating signal. This section gives an overview of two further techniques, strain monitoring and acoustic emission, which could be implemented via the same piezoelectric sensors and system infrastructure used for the previous two methods presented, to detect damage passively without the use of actuators.

In the first of these tests, a narrow coupon specimen was tested in tension, to assess the accuracy of the piezoelectric sensors for the measurement of strain by contrasting them to foil-gauge results, shown in Figure 3. The piezo data was nonlinear however, which can be attributed to thermoplastic tape that attached the sensor to the specimens. A second test was performed on a laminated plate in order to explore using piezo sensors to monitor damage events using acoustic emission. Piezo patches were affixed in the center of each of the sides along the perimeter of the specimen, and data was collected at $50 \mathrm{~Hz}$ while a graphite pencil tip was broken in several locations on the laminate. The voltage results 
from these tests are plotted in Figure 4, and from these plots one can see the acoustic event within the signal by the spikes present. At this data sampling rate however, it was not possible to resolve the arrival times of the voltage spikes accurately enough to perform a triangulation calculation. A reasonable prediction of the pencil break site could be made from wavelet plots though, by comparing the magnitude of the energy present for each piezo at the time of the breakage.

\section{Discussion}

While conclusive results were not obtained from either of the tests performed during this portion of research, along with results that have been presented in the literature these tests have proved the feasibility of implementing other damage detection methods within the infrastructure of sensors that were used for the frequency response and Lamb wave methods. Using strain monitoring methods, measuring the peak strain witnessed at the surface of a laminate could help to make a prediction of failure based upon the strain limitations of the material. Several researchers in the literature have successfully fabricated piezoelectric based strain gauges that are viable for acceptable strain rates and ranges. Additional research would have to be performed to find a more appropriate attachment mechanism for this method to be successful. Similarly, the literature has presented prior successful acoustic emission work that has been performed using sampling rates between $300 \mathrm{kHz}$ and $3 \mathrm{MHz}$ with optimized sensors. To monitor continuously, custom software would have to be used to collect and purge small buffered series of data at high acquisition rates to avoid collecting a large volume of data. Regardless, acoustic emission methods have shown the potential to provide valuable information to the system concerning the occurrence of an impact event and proximity to the sensor. Coupled with the results presented in the literature, this preliminary data demonstrated the possibility that the piezoelectric sensors could passively collect useful data with some additional software and data processing capabilities.

\section{COMPONENTS OF AN SHM SYSTEM}

This section delineates the relevant considerations and components necessary for a complete SHM system. The focus of the previous chapters of this thesis has been on the sensors used for the actual damage detection in the structure, however perhaps of equal importance and challenge are the other components used to convert, process and transmit the data from these sensors.
An overall SHM system architecture is necessary to integrate strategically all of the other components for the most efficient operation. The requirements of the end users are incorporated into the architecture in order to define the types of damage to be monitored, the critical flaw size, the weight and power budget for the system, and the level of importance of the various structural members that need to be monitored. A SHM system could operate in real-time continually monitoring a structure during operation, or discontinuously with that data can only be accessed post-operation. Next, characteristic damage signatures must be known for the particular material and structure to select appropriate sensors and sensing methods. Sensors can be used to record variables such as strain, acceleration, sound waves, electrical or magnetic impedance, pressure or temperature. In the literature it has been estimated that a SHM system for an aerospace vehicle would require between 100 and 1000 sensors, depending on its size and desired coverage area ${ }^{10}$. These sensing systems can generally be divided into two classes: passive ones that operate by detecting responses due to perturbations of ambient conditions, and active sampling systems that require externally supplied energy in the form of a stress or electromagnetic wave to properly function. Passive techniques tend to be simpler to implement and operate within a SHM system and provide useful global damage detection capabilities, however generally active methods are more accurate in providing localized information about a damaged area. Good references for selection of actuators for various active systems can be found in a review paper in the literature ${ }^{19}$.

The data from the sensors must be communicated between the sensors and to a processing unit. Some of these transfers would only require local wiring, while other may need wireless transmission. Local processors would directly interface with the sensors providing instructions and acquiring data, while a global processor would use algorithms to interpret this data and decide what actions should be taken. Most of these components require power to function, which becomes difficult when there are many components distributed throughout the surface of the structure. Power could be supplied locally by batteries or energy harvesters, or provided from within the vehicle via an electrical bus or transmissions to an inductive loop. Lastly, some systems may require an intervention mechanism as well to mitigate further damage actively, or possibly even temporarily or permanently repair the damage site. All of these components must work together to gather the information about damage in the structure, and flawlessly relay this information to an operator for the system to work reliably. 


\section{RECOMMENDATIONS FOR SHM}

The main focus of this research was to provide design recommendations and guidelines for the implementation of a structural health monitoring system in a composite structure. A successful design will use several different sensing methods, taking advantage of both the strengths and weaknesses of each. Using the sensor trade spaces shown in Figure 5 and Figure 6 a SHM designer could determine appropriate sensing methods based on the required damage resolution and power budget. It can be seen that they are all generally capable of detecting the same size of damage and can be implemented with similar size and power sensors, however frequency response and Lamb wave techniques are the only ones that can offer full surface coverage for a $1 \mathrm{x} 1 \mathrm{~m}$ plate. While some other methods, such as eddy currents, can offer better damage resolution, they are only capable of detecting damage directly below the sensor, which would drive the system to use either very large sensors or a large volume of sensors. An estimate could also be made for sensor density based on desired coverage area using the equations presented in previous papers ${ }^{13-17}$. The trade between redundancy and reliability is essential since missed damage or false-positives could prove financially fatal. Using event-driven processing, such as a passive system triggering a dormant active one could reduce power and complexity, and further gains could be reached by using ambient conditions to provide power or actuation. Lastly, it would be advantageous to design a system that was flexible enough to be retrofitted into existing aging systems.

A design proposed by the authors would use relatively small $\left(0.25-1.0 \mathrm{~m}^{2}\right)$ autonomous sensor patches as its key elements. These patches would include multiple piezoelectric sensors around their perimeter, local wiring between the sensors (longest length of $0.5 \mathrm{~m}$ ), a data acquisition/processing device (capable of sampling around $1 \mathrm{MHz}$ ), a rechargeable polymer battery with an inductive coil for power reception (50 $\mathrm{mW}$ required to power all components), and a short range wireless device (10 m transmission range). All of these components would be embedded or deposited onto a conformable insulating polymer sheet with a thermoplastic adhesive backing, so the patch could be removed if it were damaged or if the structure required repair. These patches would be generic so that they could be placed in any region of concern on a vehicle. Other sensor types could possibly be deposited onto the polymer as well in certain regions, such as meandering wires for eddy current tests or differential parallel metal tracks for thermocouple readings. A neural network algorithm could be used for the sensors to learn the topology of the area of structure they are adhered over, to collect a small database of the undamaged state, and to discern where each patch was in spatial coordinates of the structure. In operation the sensors would passively collect strain and acoustic emission data, passing their data along to their local processing units. When abnormal data is encountered, active transfer function frequency response and Lamb wave methods would be initiated, using the same piezoelectric sensors, to verify the presence of damage. Once damage is located within the patch region, the nearest neighbor patches would be contacted wirelessly to attempt to confirm the damage. This compiled, consolidated and compressed data would then be passed patch to patch to the central processing unit to be interpreted, and the damage type, severity and location would be indicated to the operator and ground crew on a computer terminal along with suggested actions. This system would function continuously during operation, and could also be automatically accessed by the operator or ground crew to perform a mid-air or ground inspection on demand. As a first step towards acceptance of such a system, the operator could rely on it only to speed ground inspections by accessing the in-situ sensor patches via an ethernet connection to replace teardown inspections.

\section{CONCLUSIONS}

Structural health monitoring systems will be an important aspect of future aerospace vehicles in order to reduce their life-cycle costs. The present research has analytically and experimentally explored potential sensing methods for damage detection. Modal analysis and Lamb wave techniques have been of particular interest since they provide sensitive and reliable indications of the presence of damage over a large coverage area. Moreover, these methods can be implemented with lightweight and conformable piezoelectric sensors that can be used to perform other methods simultaneously, such as acoustic emission and strain monitoring. To bring SHM systems to fruition however, several of the other components described above need to be researched further. Future research will also need to be performed at the architectural level to integrate these components together efficiently and on complex structures. To decide between architectural schemes, a designer will have to compare the cost of development, the cost of implementation, the cost of operation, and the impact to the production of the vehicle with the estimated savings in inspection and maintenance from traditional methods and the reliability and longevity gains. SHM systems will reduce vehicle life-cycle costs by minimizing routine inspections, averting both underuse and overuse, and predicting failure in time for preventative care. Structural heath monitoring systems are likely to be an important component in future designs of air and space-craft and in-situ piezoelectricbased NDE techniques will likely play a vital role. 


\section{REFERENCES}

1. Hall S.R. and T.J. Conquest. "The Total Data Integrity Initiative-Structural Health Monitoring, The Next Generation." Proceedings of the USAF ASIP, 1999. $2^{\text {nd }} \mathrm{ed}$.

2. Neumair M. "Requirements on Future Structural Health Monitoring Systems." Proceedings of the 7th RTO Meetings, May 1998.

3. Zimmerman D.C., Simmermacher T. and M. Kaouk. "Model Correlation using Frequency Domain Measurements." AIAA Journal, 1995, 3318-3325.

4. Abdelghani M., Goursat M. and T. Biolchini. "On-Line Modal Monitoring of Aircraft Structures under Unknown Exication." Mechanical Systems and Signal Processing, v.13, 1999, 839-853.

5. Dunne J.P., Pitt D.M. and D.A. Sofge. "Recent Advances in Active Damage Interrogation." Proceedings of the 42nd AIAA SDM Conference, Seattle, WA, 2001.

6. Giurgiutiu V., Bao J. and W. Zhao. "Active Sensor Wave Propagation Health Monitoring of Beam and Plate Structures." Proceedings of the 8th SPIE Symposium on Smart Structures and Materials, Newport Beach, CA, 2001.

7. Dalton R.P., Cawley P. and M.J.S. Lowe. "The Potential of Guided Waves for Monitoring Metallic Aircraft Fuselage Structure." Journal of Nondestructive Evaluation, v.20, 2001, 29-46.

8. Schoess J.N. "Distributed System Architecture Alternatives for Condition Based Maintenance." Honeywell Technology Center Report, 1999.

9. Van Way C.B., Kudva J.N. and Schoess J.N. "Aircraft Structural Health Monitoring System Development-overview of the Air Force/Navy Smart Metallic Structures Program." Proceedings of the SPIE Symposium on Smart Structures and Materials, San Diego, CA, 1995.

10. Marantidis C., Van Way CB. and J.N. Kudva. "Acoustic-Emission Sensing in an On-Board Smart Structural Health Monitoring System for Military Aircraft." Proceedings of the SPIE Conference on Smart Structures and Integrated Systems, v. 2191, 1994, 258-264.

11. Kessler S.S., Spearing S.M., Atalla M.J., Cesnik C.E.S. and C. Soutis. "Damage Detection in Composite Materials using Frequency Response Methods." Proceedings of the SPIE's $8^{\text {th }}$ International Symposium on Smart Structures and Materials, 48 March 2001, Newport Beach, CA, NDE 4336-01.

12. Kessler S.S., Spearing S.M., Atalla M.J., Cesnik, C.E.S. and C. Soutis. "Structural Health Monitoring in Composite Materials using
Frequency Response Methods." Accepted for publication by Composites Part B, June 2001.

13. Kessler S.S. "Piezoelectric-Based In-Situ Damage Detection of Composite Materials for Structural Health Monitoring Systems." Massachusetts Institute of Technology, Ph.D. thesis, January 2002.

14. Kessler S.S., Spearing, S.M. and C. Soutis. "Damage Detection in Composite Materials using Lamb Wave Methods." Proceedings of the American Society for Composites, 9-12 September 2001, Blacksburg, VA.

15. Kessler S.S., Spearing S.M. and C. Soutis. "Optimization of Lamb Wave Methods for Damage Detection in Composite Materials." Proceedings of the $3^{\text {rd }}$ Workshop on Structural Health Monitoring, 12-14 September 2001, Stanford University.

16. Kessler S.S., Spearing S.M. and C. Soutis. "Structural Health Monitoring in Composite Materials using Lamb Wave Methods." Submitted to Smart Materials and Structures, July 2001.

17. Kessler S.S., and S.M. Spearing. "Damage Detection in Built-Up Composite Structures using Lamb Wave Methods." Submitted to Journal of Intelligent Materials Systems and Structures, December 2001.

18. Strang G. and T. Nguyen Wavelets and Filter Banks. Wellesley-Cambridge Press, Wellesley, Ma, 1996.

19. Huber J.E., Fleck N.A. and M.F. Ashby. "The Selection of Mechanical Actuators based on Performance Indices." Proceedings of the Royal Society of London, 1997, 2185-2205. 


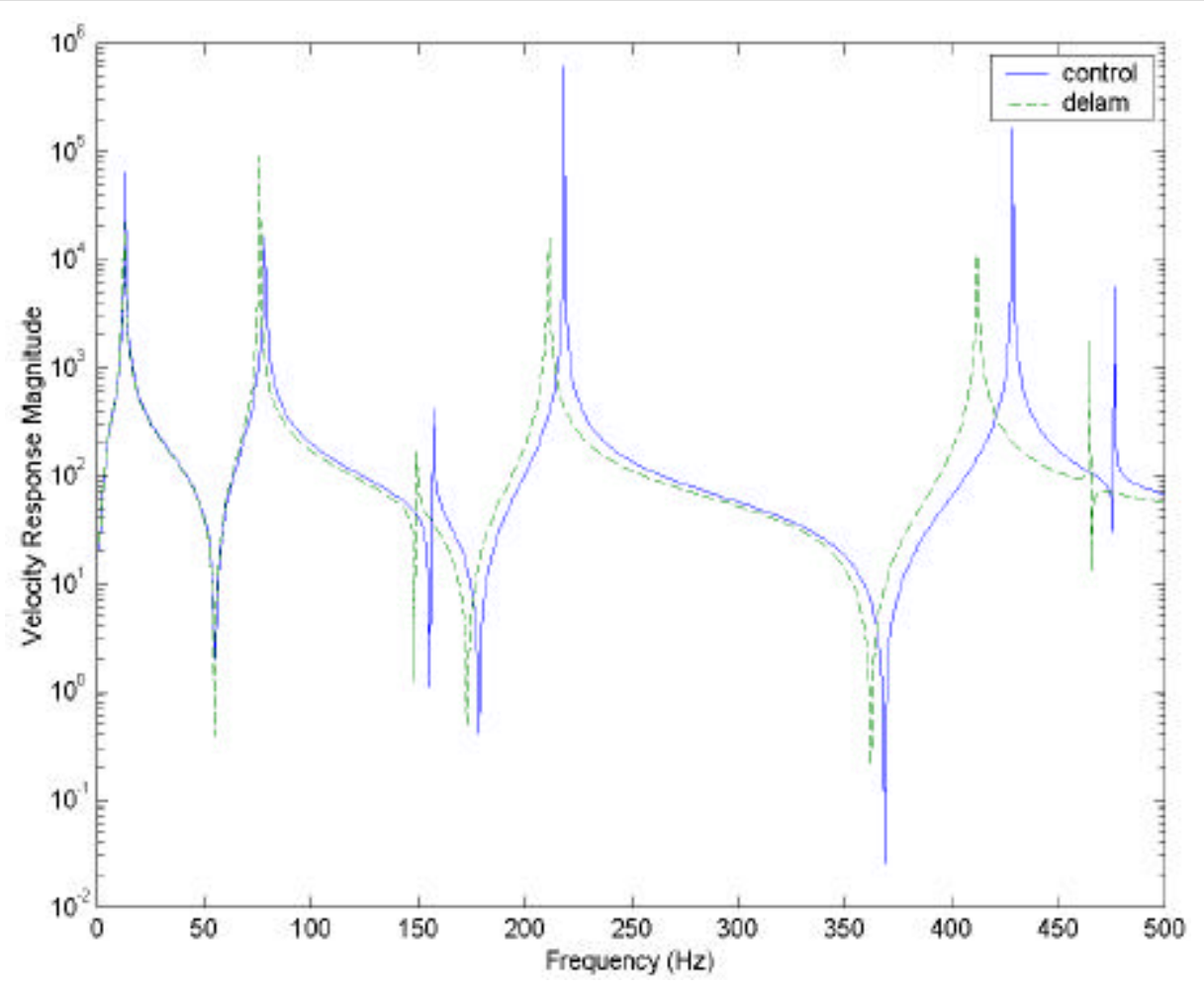

Figure 1: Frequency response transfer function plot from I-DEAS, range of $0-500 \mathrm{~Hz}$

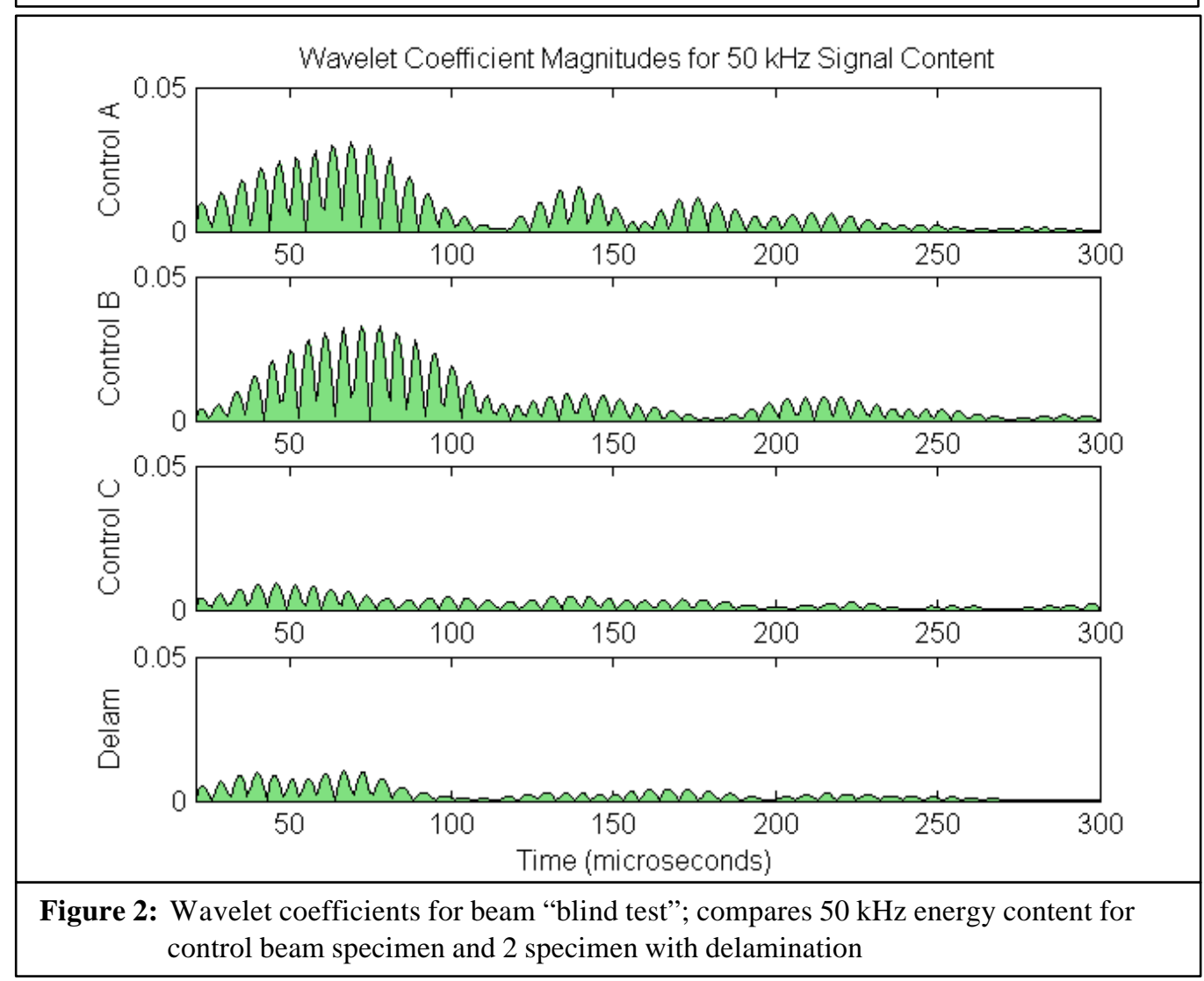




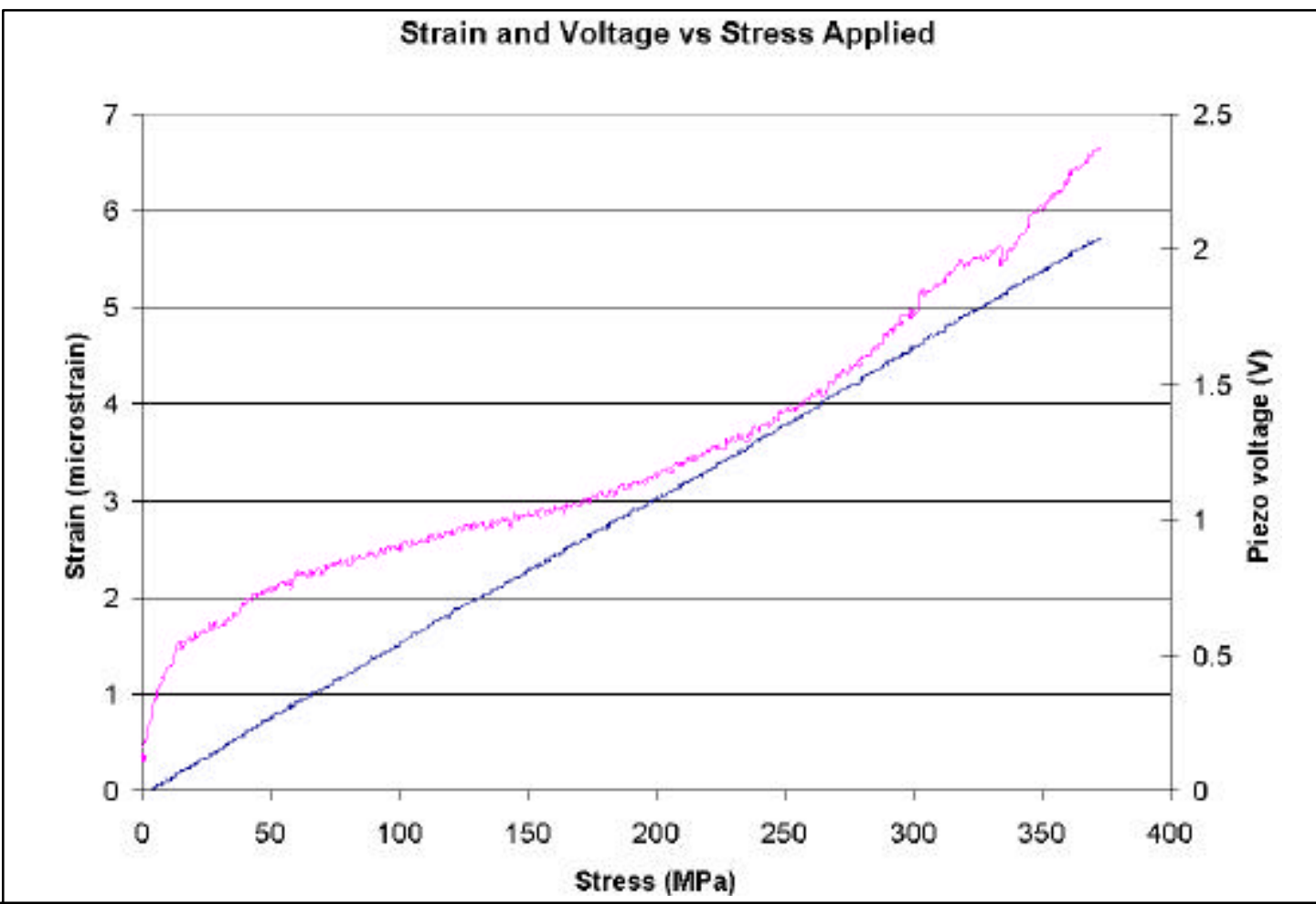

Figure 3: Rotated stress-strain plot for coupon with hole, piezo voltage data superimposed

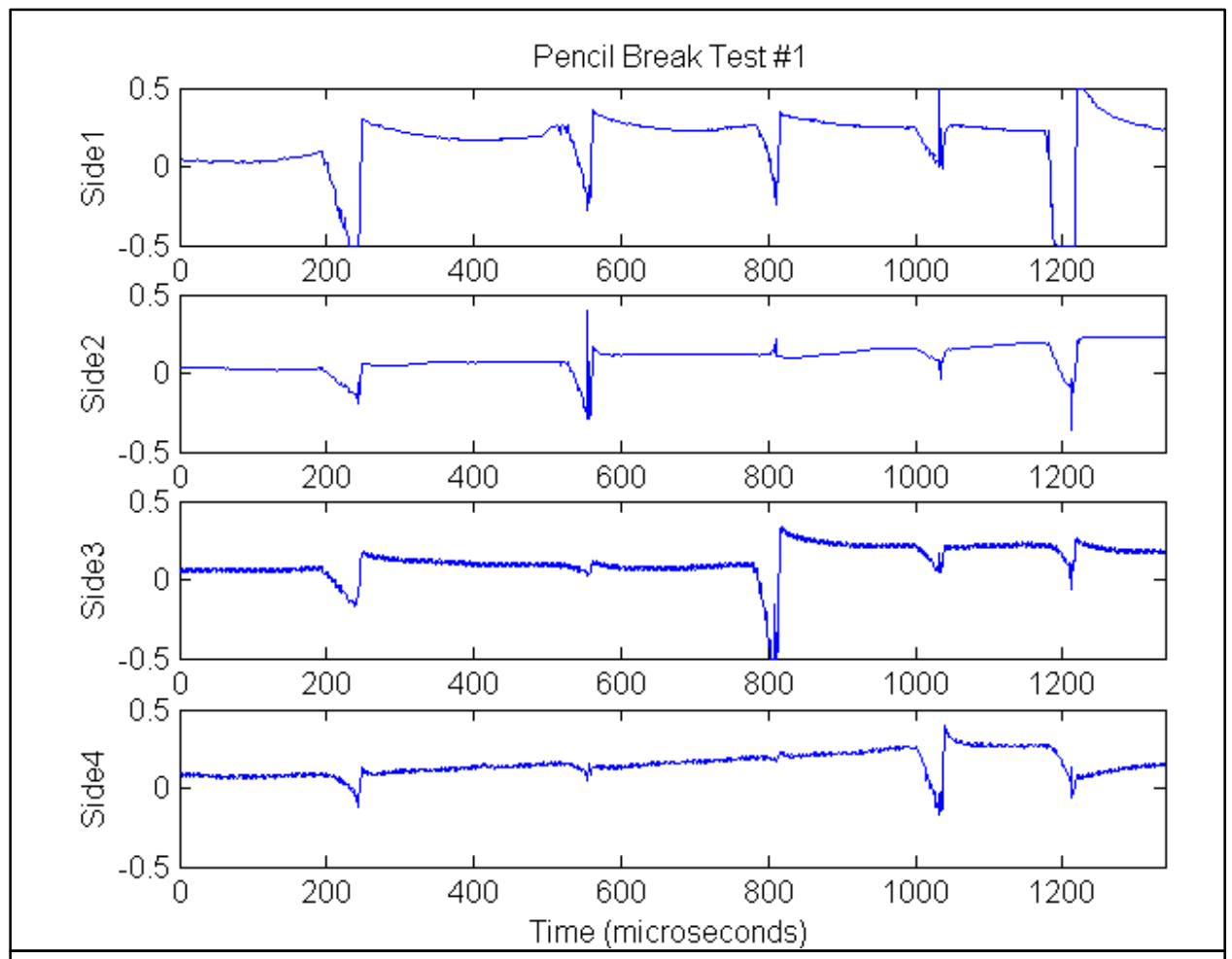

Figure 4: Time-trace of voltage signal recorded by each piezo for tests \#1 and \#2

American Institute of Aeronautics and Astronautics 


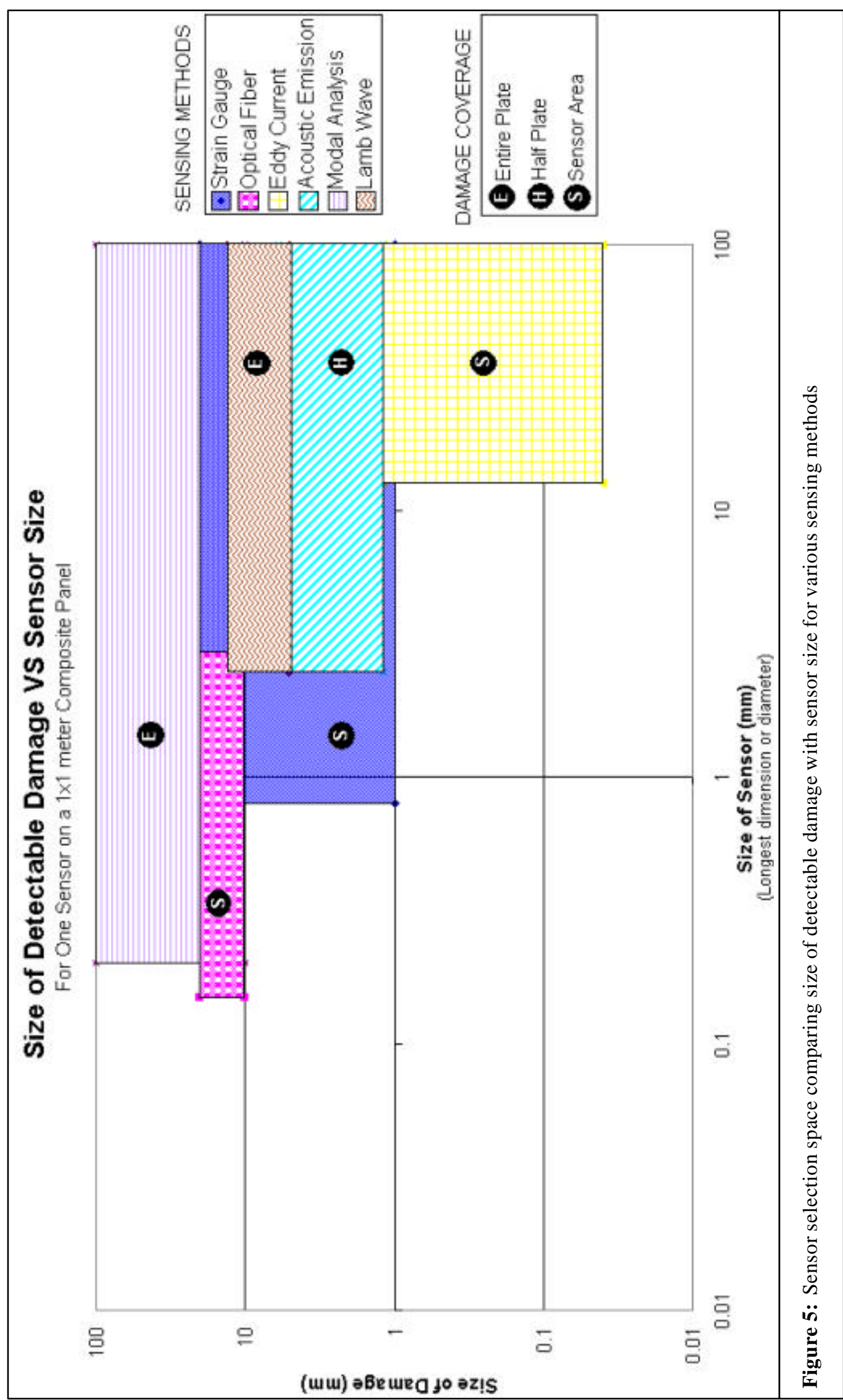




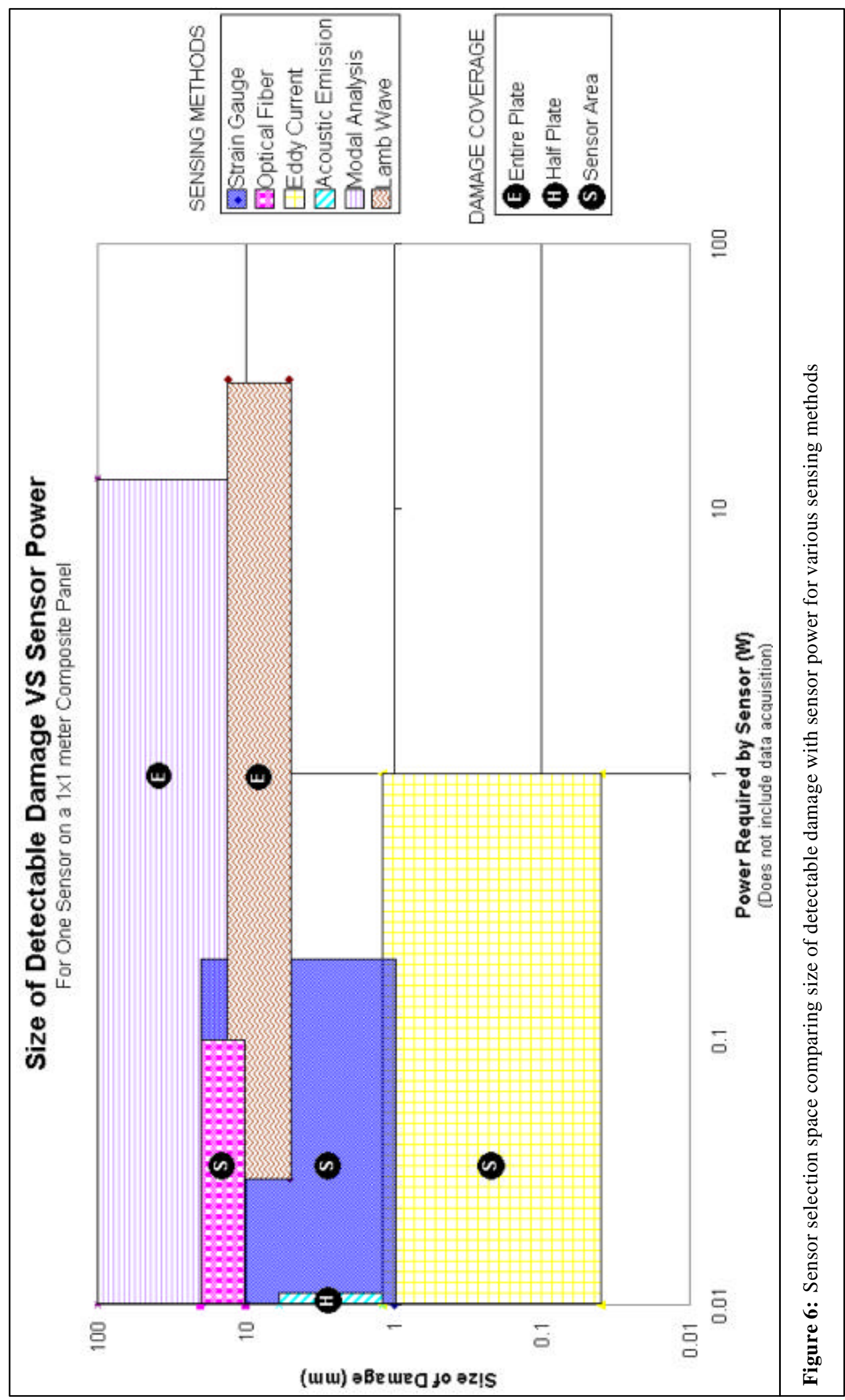


Table 1: Natural frequencies and mode shapes as determined from scanning laser vibrometer data

\begin{tabular}{|l|c|c|c|c|c|c|c|}
\hline$($ All Hz) & Shape & Control & Hole & Impact & Delamination & Fatigue & Bend \\
\hline Mode 1 & $1^{\text {st }}$ Bending & 12.5 & 12.5 & 12.5 & 12.5 & 12.5 & 12.5 \\
\hline Mode 2 & $2^{\text {nd }}$ Bending & 78.1 & 78.1 & 76.5 & 78.1 & 75.0 & 76.3 \\
\hline Mode 3 & $1^{\text {st }}$ Torsion & 157 & 148 & 147 & 137 & 146 & 137 \\
\hline Mode 4 & $3^{\text {rd }}$ Bending & 218 & 217 & 216 & 215 & 209 & 214 \\
\hline Mode 5 & $4^{\text {th }}$ Bending & 423 & 423 & 423 & 428 & 413 & 423 \\
\hline Mode 6 & $2^{\text {nd }}$ Torsion & 461 & 453 & 453 & 451 & 428 & 432 \\
\hline
\end{tabular}

Table 2: Natural frequencies and mode shapes as determined from FEM in I-DEAS

\begin{tabular}{|l|c|c|c|c|c|c|c|}
\hline All Hz) & Shape & Control & Hole & Impact & Delamination & Fatigue & Bend \\
\hline Mode 1 & $1^{\text {st }}$ Bending & 12.5 & 12.4 & 12.5 & 12.1 & 12.1 & 12.3 \\
\hline Mode 2 & $2^{\text {nd }}$ Bending & 77.8 & 77.2 & 77.5 & 75.5 & 73.7 & 76.3 \\
\hline Mode 3 & $1^{\text {st }}$ Torsion & 157 & 155 & 156 & 149 & 150 & 154 \\
\hline Mode 4 & $3^{\text {rd }}$ Bending & 218 & 217 & 217 & 211 & 213 & 216 \\
\hline Mode 5 & $4^{\text {th }}$ Bending & 428 & 425 & 426 & 412 & 413 & 422 \\
\hline Mode 6 & $2^{\text {nd }}$ Torsion & 476 & 473 & 474 & 465 & 466 & 472 \\
\hline
\end{tabular}

Table 3: Lamb wave times of flight and group velocities for narrow coupons as observed experimentally

\begin{tabular}{|l|c|c|c|c|c|}
\hline $\begin{array}{c}\text { (times in microseconds, } \\
\text { velocities in m/s) }\end{array}$ & $\begin{array}{c}\text { TOF based on } \\
\text { initial arrival }\end{array}$ & $\begin{array}{c}\text { TOF based on } \\
\text { peak arrival }\end{array}$ & $\begin{array}{c}\text { Cg based on } \\
\text { initial arrival }\end{array}$ & $\begin{array}{c}\text { Cg based on } \\
\text { peak arrival }\end{array}$ & $\begin{array}{c}\Delta \mathrm{t} \text { from } \\
\text { undamaged }\end{array}$ \\
\hline Undamaged & 216 & 218 & 952 & 944 & - \\
\hline Center cracked region & 238 & 233 & 864 & 883 & 22 \\
\hline Center 5mm hole & 226 & 230 & 910 & 894 & 10 \\
\hline Center 50x50mm delam & 261 & 258 & 788 & 797 & 45 \\
\hline Side 50x25mm delam & 231 & 220 & 890 & 935 & 15 \\
\hline
\end{tabular}

Table 4: Lamb wave times of flight and group velocities for narrow coupons as observed from FEM solutions

\begin{tabular}{|l|c|c|c|c|c|}
\hline $\begin{array}{c}\text { times in microseconds, } \\
\text { velocities in m/s) }\end{array}$ & $\begin{array}{c}\text { TOF based on } \\
\text { initial arrival }\end{array}$ & $\begin{array}{c}\text { TOF based on } \\
\text { peak arrival }\end{array}$ & $\begin{array}{c}\text { Cg based on } \\
\text { initial arrival }\end{array}$ & $\begin{array}{c}\text { Cg based on } \\
\text { peak arrival }\end{array}$ & $\begin{array}{c}\Delta \text { t from } \\
\text { undamaged }\end{array}$ \\
\hline Undamaged & 230 & 230 & 894 & 894 & - \\
\hline Center cracked region & 231 & 231 & 891 & 891 & 1 \\
\hline Center 5mm hole & 237 & 231 & 868 & 891 & 7 \\
\hline Center 50x50mm delam & 306 & 280 & 672 & 735 & 76 \\
\hline Side 50x25mm delam & 292 & 354 & 704 & 581 & 62 \\
\hline
\end{tabular}

